\title{
SULL'EFFETTO ELETTROMAGNETICO DIRETTO DI EMITTORI ALTERNATIVI IN UN TERRENO OMOGENEO
}

\author{
A. Belluigi
}

In: "Sui campi geoelettromagnetici a hassa frequenza " (1) risolvemmo una serie di prohlemi connessi con l'impiego dei campi magnetici nella prospezione greoelettrica a c.a. a hassa frequenza.

Ritorniamo qui su questioni riguardanti invece la conoscenza dei campi geoelettrici, tanto più discordi pareri vertono sull'argomento (Lewis, Horton, Wolf, Evjen, Müller).

Nella teoria del dipolo orizzontale alternativo (d.a.), pulsazione: (1) $=2 \pi f$, con l'asse lungo $x$, sulla superficie d'un suolo omogeneo lo a stratificazione orizzontale), Sommerfeld mostrò che le condizioni maxwelliane di passagrgio delle componenti e.m. (nel piano di separazione: aria-suolo di conduttivita : $\sigma$ l si soddisfano se si ammette un potenziale-vettore $m$ (p.v.). $A$, la cui componente $A_{y}$ sia identicamente nulla nell'aria e nel suolo, mentre le altre due componenti, diverse da zero, si calcolano ora agevolmente mercè grli integrali Fock-Foster [1].

W. B. Lewis ba voluto vedere [2] un conflitto tra le soluzioni Sommerfeld e quelle derivanti dall'equazione delle onde non priva dell'addendo con la costante dielettrica: [3].

Senonché quest'ultime, riferibili ad un oscillatore dipolare nello spazio vuoto, dove al posto delle velocità della luce $c$ si sostituisce la velocità complessa $c(u+j k)$, (con $n$ costante di fase, $k$ coefficiente d'estinzione del mezzol, comporta necessariamente componenti di $\bar{A}$ tutte diverse da zero.

Se la teoria di Sommerfeld prescinde in partenza, nell'equazione delle onde, dalla costante dielettrica $\varepsilon$, quella di Smith-Horton-Lewis, [3], resta pur sempre molto approssimata, trascurando essa ad un certo punto la $\varepsilon$, limitazione però che qui rimuoviamo facilmente.

A parte il fatto che la teoria di Horton non considera nell'equazione delle onde, l'addendo (non nullo per la conduzione prevalentemente jonica dei suoli, della divergenza del vettore elettrico, e ritiene $\sigma e d=$ indipendenti dalla frequenza (quindi anchessa ha sempre una consistenza molto approssimata), l'eliminazione poi nelle risolventi della $\varepsilon$ porta a limitare i risultati adeguandoli quasi del tutto ai vecchi. 
Rimuovendo, al contrario, tale approssimazione, con: $(\varepsilon \neq=0)$, si potranno dedure ulteriori, notevoli proprieti dei c.e.m. terrestri, come verrà qui tutto mostrato.

క I. - Dalle espressioni integrali delle componenti il p.v. $\bar{A}$, nell'aria e nel suolo:

$$
A_{x} \neq 0, A_{y}=0, A_{z}=0, d i v=\partial A_{x} / \partial x+\partial A_{n} / \partial z
$$

poste sotto forma di funzioni esponenziali e cilindriche con argomento la "distanza numerica " merce wli integrali Fock-Foster, si ottengono subito le 6 componenti del campo e.nu., in particolare quella orizzontale del campo elettrico parallela al dipolo, alla superficie del suolo omogeneo :

$$
E_{\mathrm{x}}=-i \omega A_{\mathrm{v}}+\frac{\partial}{\partial x}(\operatorname{div} \cdot A)+\pi \sigma=\left[2-3 x^{2} r-(1+\gamma r) c-\gamma r \mid 2 \pi \sigma r^{3}\right.
$$

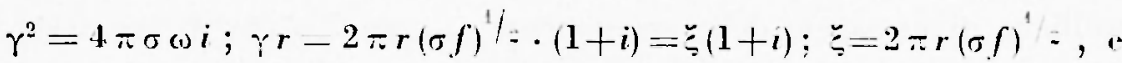

la complanare perpendicolare al dipolo che si riduce (fatto smentito però dall'esperienza), ad un valore stazionario:

$$
F_{y}=\frac{\partial}{\partial y}(\operatorname{div} \cdot A) / 4 \pi \sigma=-3 x y / r^{\prime \prime}(2 \pi \sigma)
$$

Daltronde questa deduzione, legata ai presupposti teorici di Sommerfeld, ha pure un chiaro significato fisico: la $[2]$ equivale alla classica:

$$
\begin{aligned}
& E_{x}=I d s\left[-P(x)+\frac{\partial^{2}}{\partial x^{*}} Q(x)\right], \text { con: } \\
& P(x)=\frac{i a}{2 \pi x}\left[1-(1+\gamma x) e^{-\gamma \times}\right] /(\gamma x)^{2} \simeq \frac{i \omega}{4 \pi x}\left[1-\frac{2}{3}(\gamma x)+\frac{1}{4}(\gamma x)^{2}\right] \\
& Q(x)=1 / 2 \pi \sigma x, \gamma^{2}=i(1) \sigma, Q(r)=1 / 2 \pi \sigma r .
\end{aligned}
$$

La [3] diventa: $E_{s}=\frac{\partial^{2}}{\partial x \partial y} Q(r)$, dove $Q(r)$ non a che una resistenza mutua in corrente continua.

Nel mezzo, viceversa, tutte e tre le componenti di $\bar{E}$ sono $\bar{E}\left(\vartheta_{i}\right)$, funzioni cioe della frequenza, con tipiche fisionomic analitiche. 
Le formole [2], [3], sono state dedotte da più A.A. a cominciare da Stefanescu, Yost, ecc.), indirettamente da A. W'olf [5] nello studio di un dipolo oscillatore a hassa frequenza sulla superficie esterna di due strati orizzontali sovrapposti.

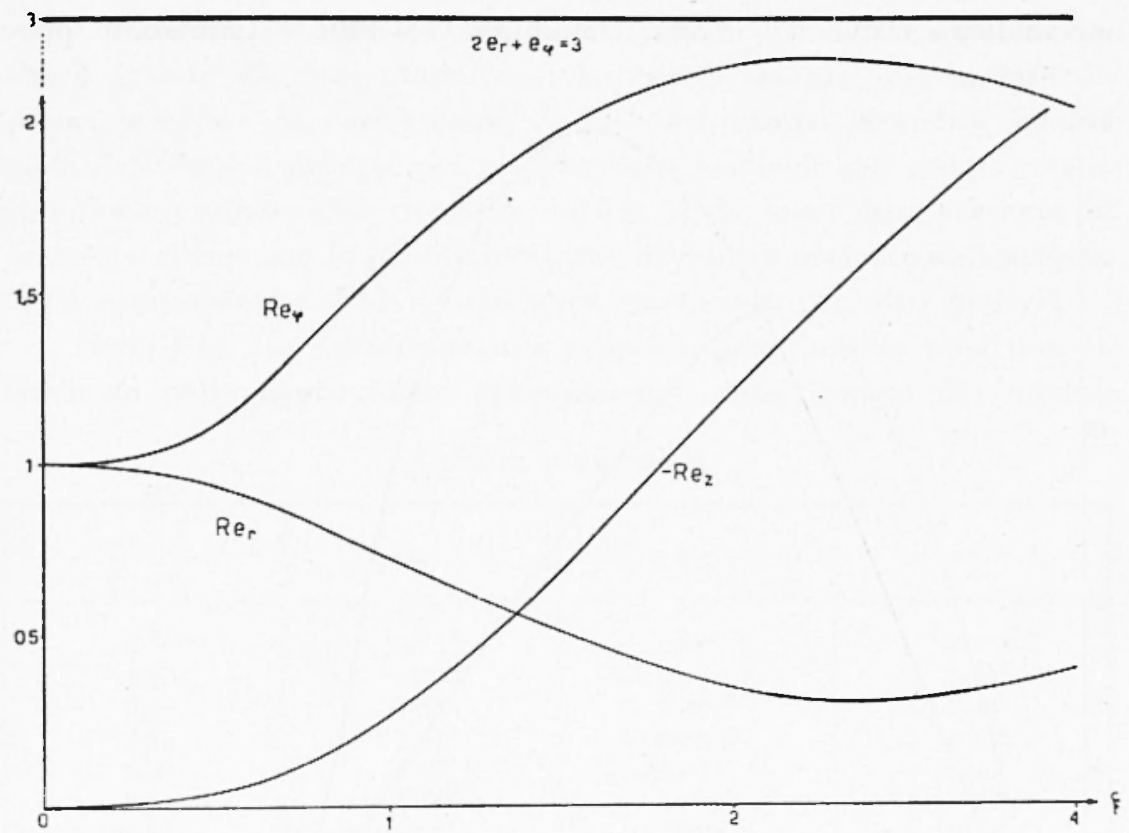

Fig. 1-a

Dalle [2], [3] si ricavano le componenti di $\bar{E}$, (radiale e tangenziale):

$F_{r}=E_{x} \cos p+E_{y} \operatorname{sen} p=-\left(\pi \sigma r^{3}\right)^{-1} \mid 1+(1+\gamma r) e^{-\gamma_{r}} j \cos p$

$E_{\mathrm{q}}=-E_{\mathrm{q}} \operatorname{sen} \varphi+E_{\mathrm{y}} \cos \varphi=-\left(2 \pi \sigma r^{3}\right)^{-1}\left[2-(1+\gamma r) e^{-\gamma_{\mathrm{r}}} \mid s \cdot n ?\right.$

quindi le "ridotte" adimensionali (v. Figc. $1_{\mathrm{a}}$ ):

$$
e_{\mathrm{r}}=E_{\mathrm{r}} / F_{\mathrm{rc}} \quad, \quad e_{\mathrm{q}}=E_{\mathrm{\varphi}} / F_{\mathrm{qc}} \quad, \quad e_{\mathrm{x}}=F_{\mathrm{s}} / E_{\mathrm{rc}},
$$

termini questi di correzione del campo statico per valori finiti di $\left(\gamma_{i}\right)$, love $E_{\text {re }}, E \varphi_{\text {c }}$, sono le componenti di $\bar{E}$ in corrente continua.

La scala delle ascisse, lineare, nella figura (1), istata ridotta a metà da $\ddot{\zeta}=2$ in poi, per rayioni di spazio. 
In Fig. 1-a appaiono solo le parti reali delle “ ridotte " del campo elettrico in funzione della "distanza numerica ». Abbiamo omesso nel disegno le parti immaginarie, inquanto, quelle delle componenti orizzontali, risultano quasi trascurabili rispetto alle reali.

E, a nostro aviso, perciò, superfluo mettere la [2] sotto una forma analitica unita: (v. Yost); comunque, volendo, $\dot{e}$ immediato pro-

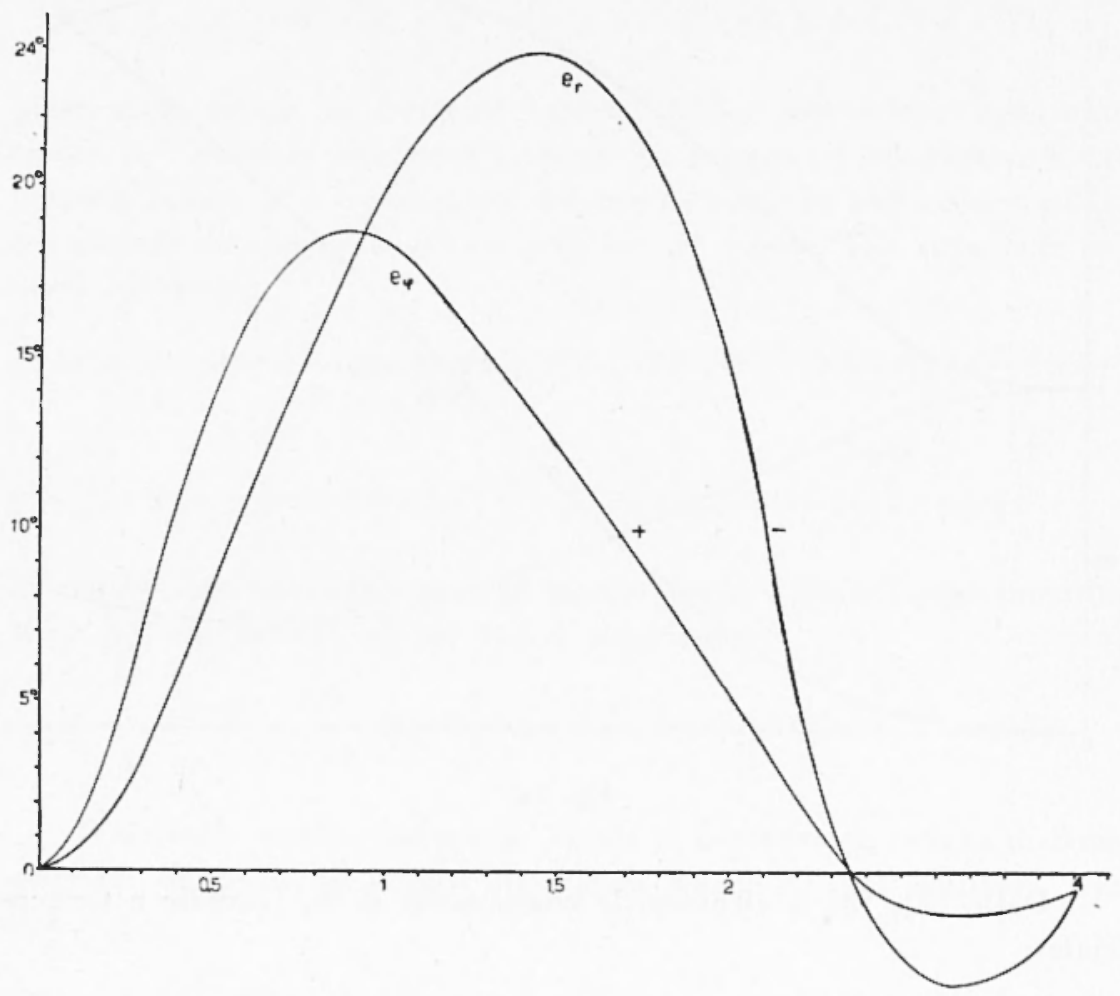

Fig. 1-b

cedere dalla [2], calcolo che non vale la pena riportare, dal quale però risalendo ai grafici si riottengono pressapoco quelli di Fị̆. 1-a.

Si vede che l'intensità del c.e. orizzontale, per piccolissime distanze numeriche dal (d.a.), è dello stesso ordine di grandezza di quella del dipolo continuo (d.c.) della stessa potenza.

Gli sfasamenti, pur su terreno omogeneo, più accentuati a distanza numerica relativamente piccola con caratteristico massimo (v. Fig. 1-l, e seguente Tabella sfasamenti), tendono a scomparire da un certo va-

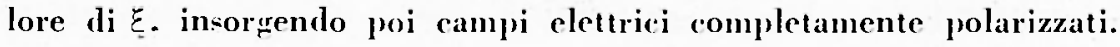


La preoccupazione dell'incidenza di questi sfasamenti, del (Yost), (nello spazio e nel tempo), accentuati nella regione più utile alle misure, può essere rimossa una volta riconosciuto il "campo di fase normale ", (relativo ai suoli omogenei), (v. Fig. 1-b), tenendone conto, quale "fattore di correzione " dei valori di campo d'osservazione.

E da osservare, per quanto almeno sugrgerisce la presente teoria, che a grande distanza numerica (che non è sempre una grande distanza metrica), la componente radiale del campo elettrico diventa metà dell'altra, e prevale poi la proiezione verticale del campo stesso. Quest'osservazione, che ricorrerà ancora, è da tener ben presente in relazione specie con le prassi americane di misure preferenziali proprio delle componenti radiali (siano pure giustificate per altri motivi).

Nella Fig. l-b) s'evidenzia una proprietà invariantiva (che non ritroviamo nelle teorie meno approssimate), della somma dei moduli

Tabella sfasamenti

\begin{tabular}{|c|c|c|c|}
\hline$\xi$ & $e_{\mathrm{r}}$ & $e_{2}$ & $e$ \\
\hline $\begin{array}{l}0 \\
0.1 \\
0.2 \\
0,3 \\
0,4 \\
0,5 \\
0,6 \\
0,7 \\
0,8 \\
0.9 \\
1 \\
1.1 \\
1.2 \\
1.3 \\
1.1 \\
1,3 \\
1,6 \\
1.7 \\
1.8 \\
1.9 \\
2.0 \\
2.2 \\
2.4 \\
2.6 \\
2.8 \\
3.0 \\
3.2 \\
3.4 \\
3.6 \\
3.8 \\
4,0\end{array}$ & 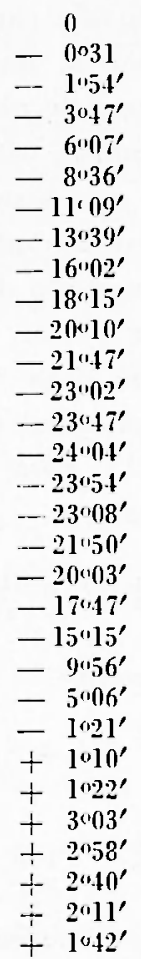 & 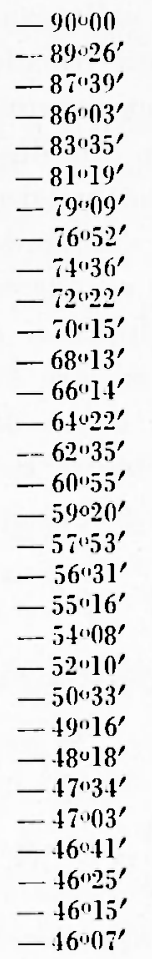 & 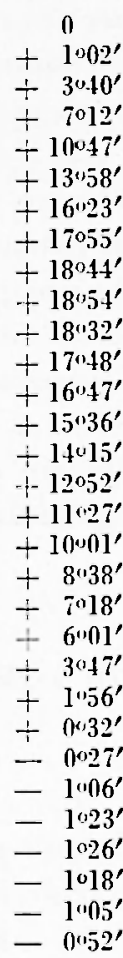 \\
\hline
\end{tabular}


delle componenti tangenziale e doppio-radiale, somma che rimane costante commque vari la "distanza numerica ": (parallela all'ascissa).

Tale " invariante" da il modo, misurata una componente, di poter subito risalire all'altra senza ulteriori misure, col presupposto (ben inteso) dellomogeneita del terreno, anzi può essere un "indice " di riconoscimento di massima di "zone anomale » se l'invariante non resta tale da punto a punto.

Se invece di considerare un dipolo e. alternativo orizzontale, si prende un dipolo e. alternativo verticale $d s$ alla superficie del suolo, per cui il campo e.m. può essere rappresentato solo dalla componente verticale del vettore hertziano, nella forma generale:

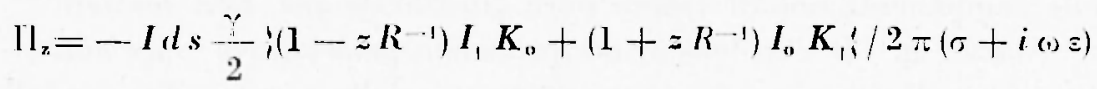

con le $I . K$. funzioni di Bessel e di Hankel modificate, di argomenti rispettivi $\left.\mid \frac{\gamma}{2}(R-z)\right],\left[\frac{\gamma}{2}, i \frac{-2}{i}\right\}$, $R$ distanza del punto d'osservazione nel suolo dallorigine delle coordinate in cui i posto il dipolo verticale, si trova per la ridotta verticale di campo elettrico Ez, un valore pure invariantivo nella grandezza di tale campo, al variare della costante numerica, retla parallela allascissa, analogamente a quella di Fig. 1-a, caratteristica dunque fondamentale elettrica del suolo uniforme, con $\Sigma=0$. t sia con dipoli orizzontali che rerticalis.

Ablbiamo messo particolarmente in evidenza tali proprieti invariantive potendo esse, valutando gli "scostamenti " nei profili, costituire il principio di un eccellente nuovo metodo di prospezione a lassa frequenza, sui cui sviluppi si riferira a parte.

$\grave{E}$ opportuno ora seguire l'evoluzione delle "componenti ridotte del c.e. m. ", tenendo conto del moltiplicatore(eim), al variare di a cominciare dalla "ridotta radiale " $[1]$. Esisa per $t=T+$ non da sfasamenti iniziali: $\overbrace{\varrho}=01$, e può scriversi:

$$
e_{\mathrm{r}}(\xi)=\frac{1}{2} e^{i \pi / 2}[1+(1+i) \xi] e^{-(1+i) \xi}
$$

con parti reale, immaginaria rispettivamente:

$$
\begin{aligned}
& R_{1}(\xi)=-\frac{1}{2}-\xi c_{0} \cos \xi-(1+\xi) \operatorname{sen} \vdots \\
& I_{1}(\xi)=\frac{1}{2} e^{-\xi}(1+\xi) \cos \xi+\xi \operatorname{son} 乡+12 \\
& R_{1}(0)=R_{1}(\infty)=0 \quad, \quad R_{1}^{\prime}(\xi)=2 \cos \omega^{\circ}-5
\end{aligned}
$$


La derivata rispetto a $\check{\Sigma}: R^{\prime}, \zeta$, si amnulla per: $\zeta=0$, cos $\vdots=0$, ciò per: $\bar{c}_{\mathrm{r}}=(2 k+1) \pi / 2$.

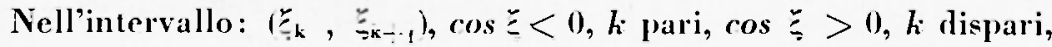
onde i punti $\sum_{-k}$ sono di massimo relativo se $k$ è pari, di minimo relativo se $k$ d dispari, rispettivamente positivi e negativi dato dhe:

$$
R_{1}\left(\zeta_{\mathrm{k}}\right)=\frac{1}{2}(-1)^{\mathrm{k}}\left[1+\frac{\pi}{2}(2 k+1)\right] e^{-(2 \mathrm{k}-1) \pi / 2}
$$

La $\bar{K}_{1}(\xi)$ parte reale di $e_{r}$, i perciò una funzione oscillante smorzata lungo $\zeta$, (con $\left.R_{1}(0)=0\right)$, per l'istante dato.

I" proprieta: Se la conduttività aumenta o diminuisce il terreno omogeneo i caratterizzato, in un dato istante, da un'onda spaziale smorzata a frequenza maggiore o minore.

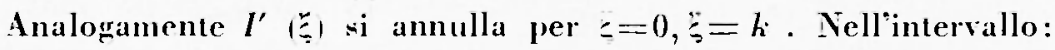
$k \pi,(k+1) \pi$, in eui : $s+n \xi>0, k$ pari, sen $\zeta<0 k$ dispari, $I_{1}^{\prime}\left(\xi_{1}\right)<0$

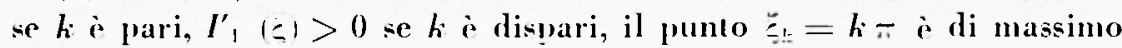
relativo $(k$ paril, di minimo relativo $(k$ disparit.

Dato che:

$$
\left.I_{1}\left(\xi_{\mathrm{k}}\right)=\frac{1}{2}\right) 11+(-1)^{\mathrm{k}} \cdot(\mathrm{l}+k \pi) e^{-\mathrm{k} \pi}
$$

i minimi relativi sono minori di l/2, i massimi maggiori di $1 / 2$.

La funzione oscillante smorzata $I_{1}(\xi)$ intorno all'asintoto: $I_{1}(\xi)$ $=1 / 2$, i sfasala rispello alla precedente $\mathrm{di} i+\pi / 2$ )

Analoghe considerazioni valgono per la "ridotta tangenziale ", al variare di 0 , sempre per $t=T+$ (valore particolarmente idoneo).

$$
\begin{aligned}
& e_{\varphi}(\xi)=\epsilon^{i \pi / 2}\left[2-(1+\xi+i \xi) e^{-(\xi+1)]}\right. \\
& R_{2}(\xi)=e^{-\zeta}[\zeta \cos \check{\zeta}-(1+\check{\xi}) \operatorname{sen} \check{\zeta}]
\end{aligned}
$$

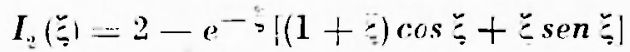

$$
\begin{aligned}
& R_{,}(0)=R_{\underline{a}}(\infty)=0 \quad, \quad R^{\prime}(\xi)=-2 \xi \cos \xi \cdot e^{-\xi}
\end{aligned}
$$

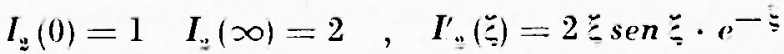

La $R^{\prime}:(\xi)$ ¿ nulla per $\Xi=0, \xi_{k}=\frac{\pi}{2}(2 k+1)$.

In $\zeta_{k}, \zeta_{k-1} \mid, \cos \check{c}<0, k$ pari, cos $\Xi>0, k$ dispari, onde $R^{\prime},(\zeta)>0$ ( $k$ paril, $\bar{K}^{\prime}:(\zeta)<0$ ( $k$ dispari), $\check{\zeta}_{k}$ è di minimo relativo se $k$ e pari, di massino relativo se $h$ è dispari. 
Inoltre:

$$
R_{\mathrm{z}}\left(\xi_{\mathrm{k}}\right)=-(-1)^{\mathrm{k}}\left[1+(2 k+1) \frac{\pi}{\underline{2}}\right] \cdot e^{-\frac{\pi}{2}(2 \mathrm{k}+1)}
$$

quindi i massini relativi sono positivi, i minimi negativi.

La $R_{2 .}(\zeta)$ ha la stessa fase iniziale e caratteristiche di $R_{1}$ (G) oscillante lungo $\check{E}$.

La $I_{2}^{\prime}(\zeta)=1$ per $\xi_{k}=k \pi$ nell'intervallo $\left(\check{\zeta}_{k}, \zeta_{k-1}\right)$, sen $\zeta_{5}>0$ se $h$ i pari, sen $\zeta<0$ se $k$ i dispari: onde in $\left(\xi_{k}, \zeta_{k-1}\right)$ si ha $I_{.}^{\prime}(\xi)>0, k$ pari, $I_{:}^{\prime}(\xi)<0, k$ dispari: segue nel punto $\ddot{\zeta}_{k}$ un minimo per $h$ pari, un massimo (relativi) per $k$ dispari.

Inoltre: $I:\left(\zeta_{k}\right)=2-e^{-k T} \cdot(1+k \pi) \cdot(-1)^{k}$, la funzione $\dot{e}$ oscillante, smorzata intorno all'asintoto $I_{\underline{.}}(\xi)=2$, con inizio da $I_{. \prime}(\xi)=1$, sfasata di $-\frac{\pi}{2}$ rispetto a $R \cdot(\xi)$.

Riassumendo: $\left[R_{1}(\xi), \zeta\right]$ ì una oscillazione smorzata intorno a : con $R_{1}(\zeta)=0$ all'origine; $\left[I_{1}(\zeta), \zeta\right]$ si smorza intorno a $I_{1}(\zeta)=1 / 2$, con inizio $I_{1}(\xi)=1 ;[R,(\zeta), \zeta]$ i del tutto simile a: $\left[R_{1}(\xi), \zeta\right]$; $\left[I_{2}(\Xi, \Xi]\right.$ in opposizione a $\left[I_{1}(\zeta), \Xi\right]$, oscillante intorno a $I_{a}(\zeta)=2$.

2"1 proprietà: Da un certo valore di $\zeta$ in poi i moduli delle ridotte radiale e tangenziale permangono costanti, e il decremento spaziale con l'ulteriore aumentare di $\zeta$ si riduce, per le componenti del campo, solo a quello relativo al dipolo a $c . c$, inversamente proporzionale al cuho della distanza metrica.

$3^{a}$ proprieta: Venendo all'approssimazione imposta dal valore altissimo delle lunghezze d'onda $\lambda$ a basse frequenze, rispetto alle normali distanze $r$ dei punti di misura dal generatore dipolare di c. a., per $\check{\zeta}=2 \pi r \lambda \ll \ll 1$, lungo l'asse dipolare (prassi seguita nelle misure) si ha: $p=0, E_{4}=0$

$$
R\left(F_{\mathrm{r}}\right)=I \cdot l \cdot \xi^{2} \pi \sigma r^{3}=4 \pi I \cdot l \cdot I / r,
$$

(dove $l$ a la lunghezza del dipoloi valore aperiodico, indipendente dalla conduttivita, che al variare del coefficiente: $1 \pi I l \cdot f$ i espresso da un fascio d'iperboli equilatere.

Nelle identiche condizioni di collinearità esterna polare, HortonLewis, (per $n=h$, trovano la stessa formola [12], ma $i$ bene subito avertire che quest"importante risultato, (di grande momento per la teoria delle riflessioni e. m. a bassa frequenza), non ¿ piu valido se 
si considera $n \neq k$, (come mostreremo nel $\$ 3$ ), risultando $-v$. formola $[36]-R\left(E_{r}\right)$ max. dipendente invece sia dalla conduttivita che dalla costante dielettrica del suolo.

$\$$ 2. - Assumiamo convenzionalmente per la conduttività media delle formazioni sedimentarie $\sigma=10^{!}$e.s.u., $\Sigma_{\mathrm{r}}$ circa 10 , permeabilita magnetica unitaria.

Se la corrente di conduzione prevale su quella di spostamento: $\Delta==16 \pi^{\prime \prime} \sigma^{2} / \omega^{2} \varepsilon^{-}>>1$, la "costante di fase " uguaglia il coefficiente d'attenuazione $(n=k=\sqrt{\sigma f}$ ), e le formole di Horton [3] che danno $E_{r}\left(\sigma>>\varepsilon^{\prime}, E_{(p}(\sigma>>\varepsilon)\right.$ perdono i termini con ( $\left.\varepsilon\right)$ :

$$
K^{\prime \prime} n^{-2}=\lim \left[\left(\Delta^{-1}+1\right)^{1 / 2}-\Delta^{-1}\right] \cdot\left[\left(\Delta^{-1}+1\right)^{1 / 2}+\Delta^{-1}\right]=1
$$

Dato questo, tanto varrebhe attenersi alla teoria di Sommerfeld che trascura " $\approx$ " in partenza, e infatti abbiamo ben dedotto la [12] che s'identifica con la formola [20] per la misura del campo elettrico radiale.

Tuttavia una sostanziale differenza tra le 2 teorie appare dal comportamento della componente orizzontale $E_{y}$, che, per la [3] è stazionaria, mentre dalle “ridotte" di Horton-Lewis:

$$
\begin{gathered}
E_{\mathrm{r}} / F_{\mathrm{rc}}=e_{\mathrm{t}}=[(1+\xi)+i \xi] \exp \cdot[-\xi+j(\omega t-\xi)] \quad[13] \\
E_{\mathrm{ff}} / E_{\mathrm{c} \mathrm{c}}=e_{\mathrm{s}}=[(1+\xi)+i \xi(1+2 \xi)] \exp \cdot[-\xi+j(\omega t-\xi)]
\end{gathered}
$$

discende che:

$$
\begin{gathered}
E_{\mathrm{y}}=E_{\mathrm{y}}(\omega)=E_{\mathrm{r}} \operatorname{sen} \varphi+E_{\mathrm{y}} \cos \varphi=\frac{I l \operatorname{sen} 2 \xi}{4 \pi \sigma r^{3}}[3(1+\xi) \cos \zeta+ \\
+\zeta(3+2 \zeta) \operatorname{sen}]\left[e^{-\xi}\right]_{\mathrm{t}=0}
\end{gathered}
$$

il che, almeno in parte, (incidendo più cause sulla componente $\left.E_{y}\right)$ ( $(\omega)$ è conforme all'esperienza (v. Lewis) (2). È opportuno perció soffermarsi su questa teoria che sarà poi da noi integrata (\$ 3 ) senza tener più conto dell'approssimazione qui fatta: $n=k$, valevole per frequen. ze molto minori di 1 megaciclo sec.

L'identificazione intanto di $n$ con $k$, (parte reale e immaginaria della costante di propagazione nella sua espressione completa, ciò con $\varepsilon$ ), porta a stabilire una velocita di trasmissione delle o.e.m.: $v=c(f / \sigma)^{1 / 2} \mathrm{~cm} / \mathrm{s} s=\left(10^{9} \mathrm{f} / \sigma\right)^{1 / 2} \mathrm{~cm} / \mathrm{sec}$, velocita che nei suoli a 
conduttivita finita cresce con la frequenza (mezzi trasmittenti perció dispersivi) con relativa attenuazione d'onda (mezzi altresi dissipativil, mentre nei non conduttori tale velociti di fase i indipendente dalla frequenza e l'onda si propaga senza distorsione.

A sua volta la lunghezza d'onda e.m. nei suoli sara:

$\left.i=c(\sigma f)^{-1 / 2}=10^{4} / \overline{10}(\sigma \cdot f)^{1 / 2}\right)\left(c m\right.$. Un'onda e.m. ad es. a $10^{i} \mathrm{p} / \mathrm{sc}$, mentre nell'aria $\dot{e}$ sui $3000 \mathrm{~m}$, in un terreno, con $\sigma=10^{-4}$ Siemens/cm, si riduce a $\lambda=100 \mathrm{~m}$, lunghezza che esprime altresi la profondita di penetrazione della ea. nel mezzo: lskin depthi.

Lal " $\lambda$ " (lunghezza d'onda) e relazionabile percio alla a $\Sigma$ " : $2 \pi r / \lambda$, in generale: $<<1$ alle hasse frequenze.

La |13], che da la "ridotta radiale" "1. ¿ scomponibile nelle sue parti reali e immaginaria:

$$
\begin{aligned}
& R_{1}(\xi)=e^{-\xi}\{(1+\xi) \cos ((1) t-\xi)-\xi \operatorname{sen}((1) t-\underline{\xi})] \\
& \left.I_{1}(\xi)=e^{-\xi} \xi \operatorname{s}(\omega) t-\xi\right)+(1+\xi) \operatorname{sen}((1) t-\xi) \mid \\
& R_{1}(0)=c s(1) t, R_{1}(\infty)=0, R_{1}^{\prime}(\xi)=2 \xi \theta-\operatorname{sen}((1) t-\xi) \\
& I_{1}(0)=\operatorname{sen}(1) t, I_{1}(\infty)=0, I_{1}(\xi)=-2 \xi e^{-\zeta \cos ((1) t-\zeta)}
\end{aligned}
$$

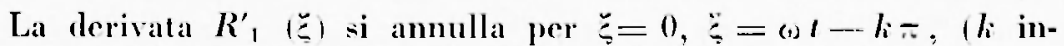
tero negativo). $R_{1}(\xi)$ presenta un minimo nei punti: $\vdots=$ (1) $1+$ $2 \pi(k+1)$, un massimo in: $\vdots=(1) l+2 k \pi$.

La parte reale $R_{1} 1 \Xi$, della componente: $r(\xi)$ i pertanto oscillatoria smorzata intorno all'ascissa $\Xi$, in dipendenza del fattore: $e^{-\vdots}$ a fase iniziale $(\Sigma=0)$ : $\cos (1) t$.

Analogamente $r_{1} ほ \xi$ si anmulla per $\check{\zeta}_{1}=0, \vdots=(1) t-\frac{\pi}{2}(2 k+1)$, ( $k$ intero newativol. Vell'intervallo $(0,-61)$ e negli intervalli: (s) $t+$ $\frac{\pi}{2}(4 k-1),(\omega) t+\frac{\pi}{2}(4 k+1)$, si ha $:-\cos \left((1) t-\frac{\xi}{3}\right)<0, I_{1}^{\prime}(\xi)<0$, $I_{1}\left(\begin{array}{l}5 \\ ) \\ \text { cerescente. }\end{array}\right.$

Negli intervalli: (1) $t+(4 k-3) \frac{\pi}{2},(1) t+(1+k-1) \frac{\pi}{2}$,

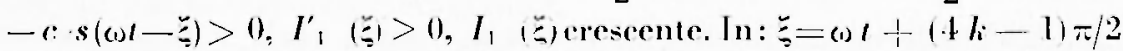
$I_{1}(\xi)$ ha un massimo, in: $\vdots=(1) t+1 / k+11 \pi / 2$ mn minimo relativo.

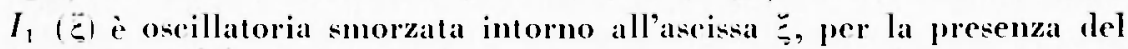
fattore: $e^{-\xi}$, a fase iniziale: $\operatorname{sen}(1) t$. 
La "ridotta tangenziale " si scompone in:

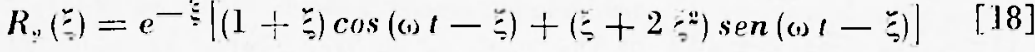

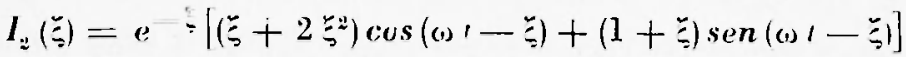

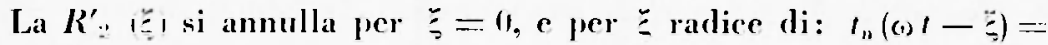
!l (1-

Pertanto le ulteriori radici di $R^{\prime}:(\check{\zeta})=0$ saranno le ascisse dei

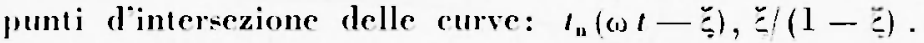

Come risulta dal grafico (v. Fig. 2a) tali intersezioni sono infi-

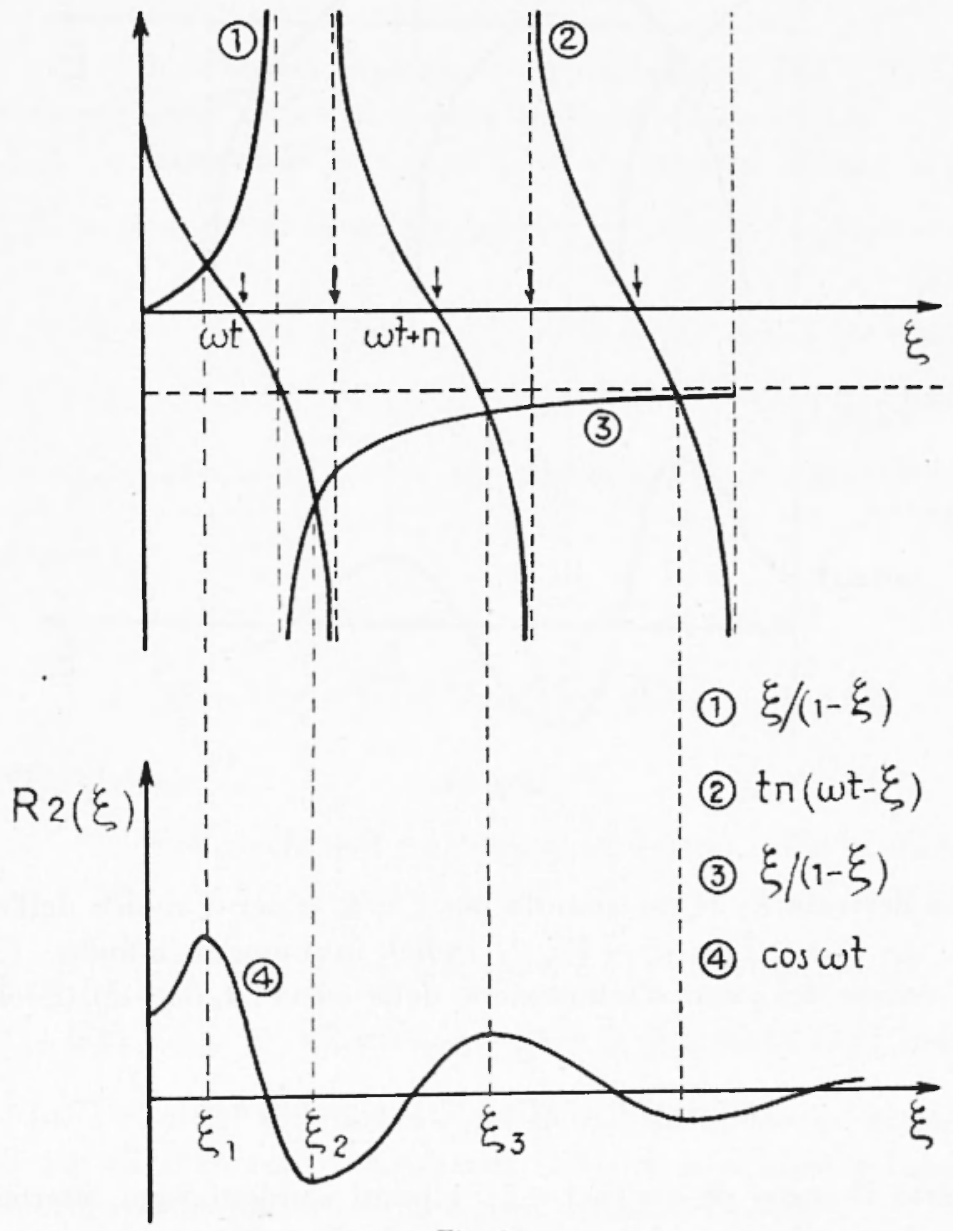

Fig. 2-a 
nite, e dette: $\xi_{1}, \breve{\zeta}_{2}, . \check{\zeta}_{k}, \ldots$ le loro ascisse, se $\breve{\zeta}_{k}>1$, si ha: $\omega t+$ $(k-1) \pi<\xi_{k}<\left\{(1) t+(2 k+1) \frac{\pi}{2}\right.$.

A causa del segno di $\cos (\omega t-\xi)$ tra $\left(\xi_{k-1}, \xi_{k}\right)$, gli $\xi_{\text {sono }}$ alternativamente di massimo e di minimo per $R_{2}(\xi)$.

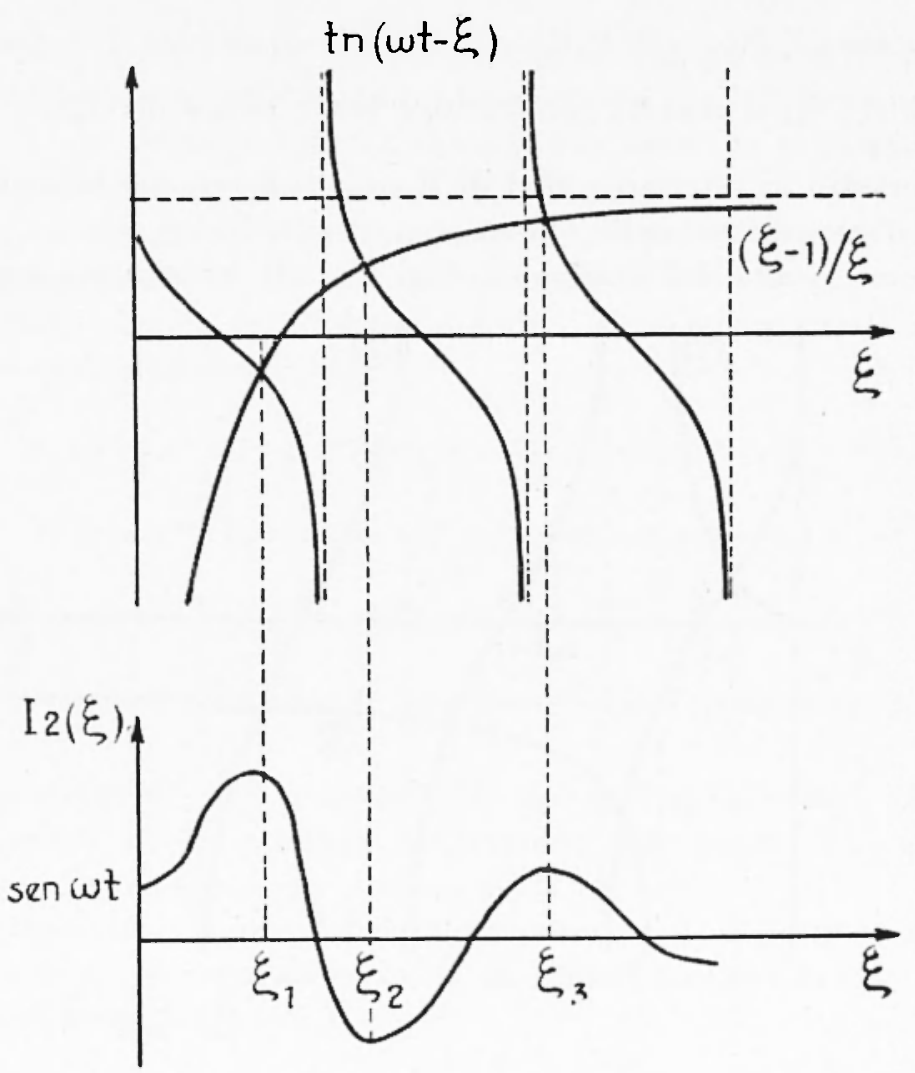

Fig. 2-b

La derivata $I_{2}^{\prime}(\xi)$ si anmulla per $\zeta=0$, e per $\zeta$ radice dell'equazione: $t_{\mathrm{n}}(\omega t-\xi)=(\xi-1) \xi^{-1}$, radici in numero infinito, $\left(\xi_{1}, \xi_{\ldots}\right.$. $\left.\zeta_{k}, ..\right)$, aseisse dei punti d'intersezione delle curve: $t_{n}(\omega t-\xi),(\xi-1) \xi^{-1}$ tali che:

$$
\text { (1) } t+(2 k-1) \pi / 2<\xi_{k}<\omega t+k \pi
$$

Dato il segno di $\cos (\omega t-\xi)$, i punti suddetti sono alternativamente di massimo e minimo relativo: (v. Fig. 2b). 
Come nella nostra teoria precedente si riscontra concordanza di fase tra le parti reali di $e_{r}$ e $e_{\varphi,}$, tra le immaginarie di $e_{r}$ e $e_{r,}$, alla loro volta in quadratura con le rispettive reali.

Lungo l'asse polare, in cui conviene effettuare le misurazioni, risultando minimizzato "l'effetto diretto " e.m. del dipolo orizzontale, sussiste solo la componente di campo radiale, conseguentemente se gli elettrodi potenziali distano dalla sorgente di corrente solo di una frazione di lunghezza d'onda, $\measuredangle<<1$, (come in generale accade), ne segue la formula approssimata di Horton-Lewis:

$$
\left(F_{\mathrm{rad}}\right) \simeq \frac{I l}{k \sigma} \cdot \xi 2 / r^{3}
$$

dove " $l$ " è la distanza dipolare, formola identica alla [12], da noi ricavata per altra via (e cioè dalla teoria di Sommerfeld).

$\S 3$. - Ritenendo ora: $n \neq k$, le componenti ridotte saranno:

$$
E_{\mathrm{r}} / E_{\mathrm{ro}}=\left[1+c^{-1} \omega r(k+j n)\right] \exp \cdot\left[-c^{-1} \omega k r+j \omega\left(t-n r c^{-1}\right)\right] .
$$

$$
\begin{array}{r}
E_{1} / E_{\mathrm{to}}=\left[1+c^{-1} \omega r(k+j n)+\left(c^{-1} \omega r\right)^{2}(k+j n)^{2} \exp \cdot\left[-c^{-1} \omega k r+\right.\right. \\
\left.+j \omega\left(t-n r c^{-1}\right)\right]
\end{array}
$$

$$
2 k^{2}=\varepsilon\left[\left(1+16 \pi^{2} \sigma^{2} /\left(\omega^{2} \Sigma^{2}\right)^{1 / 2}-1\right] ; 2 n^{2}=\varepsilon\left[\left(1+16 \pi^{2} \sigma^{2} / \omega^{2} 3^{2}\right)^{1 / 2}+1\right]\right.
$$

Posto:

$$
\begin{aligned}
& \text { (1) } k r c^{-1}=\xi \text {, } \omega n r c^{-1}=\xi_{\prime}, \xi \xi=a=n k \\
& \beta=\left(1+16 \pi^{2} \sigma^{2} / \omega^{2} \varepsilon^{2}\right)^{1 / 2}, a^{2}=(\beta+1) /(\beta-1)>0
\end{aligned}
$$

la [21] diviene:

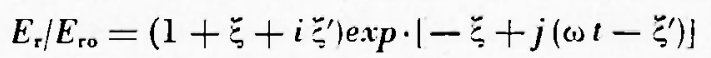

con :

$$
\begin{aligned}
& \left.R \mid E_{\mathrm{r}} / E_{\mathrm{rc}}\right]=e^{-\xi}\left[(1+\xi) \cos \left(\omega t-\xi^{\prime}\right)-\xi^{\prime} \operatorname{sen}\left(\omega t-\xi^{\prime}\right)\right]
\end{aligned}
$$

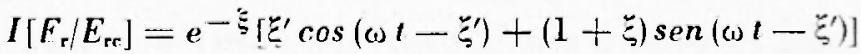

Visti i risultati sperimentali del Lewis, quelli teorici di M. Müller, Evjen ed altri, relativi ai grandi valori di $\varepsilon_{\mathrm{r}}$ a hasse e bassissime frequenze, consideriamo (se non altro a titolo d"ipotesi):

$$
\Delta=16 \pi^{2} \sigma^{2} / \omega^{2} \varepsilon^{2}=16 \pi^{2} \sigma^{2} / \omega^{2} \varepsilon_{0}^{2} \varepsilon_{r}^{2}<<1, s_{0}=0,866 \cdot 10^{-13} \text { F/cm. }
$$


Quest'assunzione che per terreni normali con: $\sigma=10^{-4} \mathrm{~S}_{\mathrm{I}_{1}} / \mathrm{cm}$ equivale porre: $\left(\varepsilon_{1}, \omega\right)>10^{*}$, si può giustificare in 2 modi: o ritenendo la costante dielettrica relativa $\varepsilon_{r}$ costante e altissime frequenze, o $\varepsilon_{r}$ altissima dell'ordine voluto da Evjen (e variabile), e basse frequenze. Nel $1^{\circ}$ caso per $\varepsilon_{\mathrm{r}}=10$ occorrono infatti frequenze di unità $10^{7}$, e viceversa per $\omega=10$ occorreranno $\Xi_{\mathrm{r}}$. dell'ordine di grandezza di unità $10^{\top}$.

Ciò permette di sviluppare $\beta$ mediante la serie binominale, e trascurare i termini di grado superiore al $1^{\circ}$ :

$$
\begin{aligned}
& \beta^{2}=1+16 \pi^{2} \sigma^{2} / \omega^{2} \varepsilon^{2}, \beta=\sim 1+8 \pi^{2} \sigma^{2} / \omega^{2} \varepsilon^{2}, a=\frac{(\beta+1)^{1 / 2}}{(\beta-1)^{1 / 2}}=\left(1+\frac{\omega^{2} \varepsilon^{2}}{4 \pi^{2} \sigma^{2}}\right)^{1 / 2} \\
& \text { [24'] } \\
& R\left[E_{\mathrm{r}} / E_{\mathrm{rc}}\right]=e^{-\zeta}[(1+\xi) c s(\omega t-a \check{\zeta})-a \dot{\zeta} \operatorname{sen}(\omega t-a \check{\zeta})]
\end{aligned}
$$

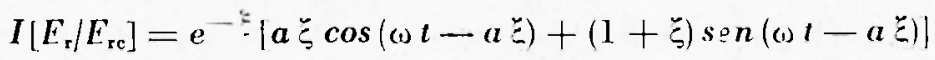

Analogamente la $[22]$ diviene:

$$
\begin{aligned}
& E_{1} / E_{\mathrm{to}}=\left(1+\xi+i \xi^{\prime}+\breve{\zeta}^{2}-\breve{\zeta}^{\prime 2}+2 i \check{\zeta} \breve{\zeta}^{\prime}\right) \exp \cdot\left|-\breve{\zeta}+i\left(\omega t-\check{\zeta}^{\prime}\right)\right|
\end{aligned}
$$

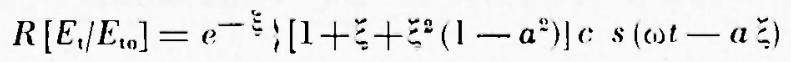

$$
\begin{aligned}
& -a \breve{\zeta}(1+2 \xi) \operatorname{sen}(\omega t-a \check{\zeta}) ! \\
& \left.I\left[E_{\mathrm{t}} / E_{\mathrm{o}}\right]=e^{-\xi} ; a \xi(1+2 \xi) \cos (\omega) t-a \xi\right) \\
& +\left[1+\xi+\xi^{2}\left(1-a^{2}\right)\right] \cdot \operatorname{sen}(\omega t-a \xi) !
\end{aligned}
$$

Fissiamo ora particolarmente l'attenzione sulla parte reale della componente radiale (prescelta nelle misure sul terreno).

Mentre nella situazione: $(n=k)$, per $t=T / 4$ si ha:

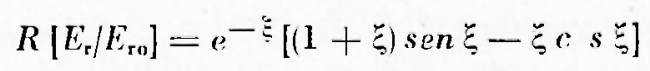

formola che s'identifica con la nostra [8]; per: $\boldsymbol{n} \neq k, \downarrow<<1$, troviano:

$$
R\left[F_{\mathrm{r}} / F_{\mathrm{ro}}\right]=e^{-\xi}[(1+\check{\zeta}) \operatorname{sen}(a \xi)-a \zeta c s(a \zeta)]
$$

Dalla componente reale radiale relativa alla situazione $\sigma^{*}>>\omega^{2} \varepsilon^{2}$ si passa a quella per: $\sigma^{*}<\left\langle(1)^{2} \varepsilon^{2}\right.$ ponendo nell'espressione di $R(E)_{r}$ al 
posto di $\zeta$ una nuova "costante numerica ): $a \zeta>\xi$, precisamente neqrli argomenti dei termini trigonometrici, e loro coefficienti (tranne per: sen $(a \xi)$, il cui coefficiente rimane invariato: $(1+\xi)$, e per: $e^{-}$tenendo conto del segno cambiato in $\cos (a \xi)$.

La: $R\left[E_{\mathrm{r}} / E_{0}\right]=R(\zeta)$, che per $t=0, a>1, \xi>0$, diventa:

$$
\begin{gathered}
R(\xi)=e^{-\xi}[(1+\xi) c \operatorname{si} \xi \xi+a \xi \sin a \xi \text { ha }: R(0)=1, R(\infty)=0, \\
R^{\prime}(\xi)=\xi e^{-\xi} \cdot\left[\left(a^{2}-1\right) \cos (a \xi)-2 a \operatorname{sen}(a \xi)\right]
\end{gathered}
$$

con derivata che si annulla per $\breve{c}=0$, tn $(a \check{c})=\left(a^{2}-1\right) / 2 a>0$.

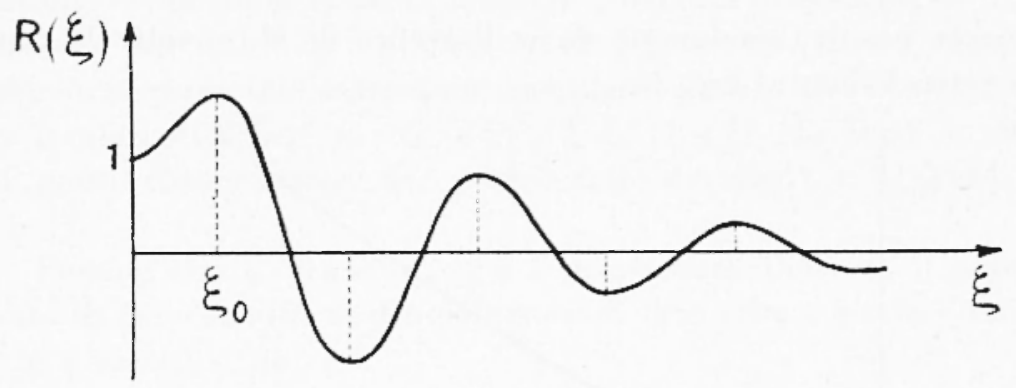

Fig. 2-c

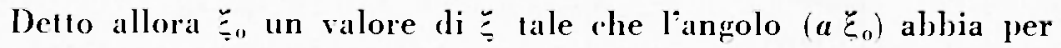
tangente: $\left(a^{2}-11 / 2 a\right.$, compreso tra $O$ e $\pi / 2$, otterremo infinite altre radiei della $R^{\prime}(\Xi)$ per tutti $\mathrm{i}$ valori di $\Xi$ tali che: $\Xi_{\zeta}=\Xi_{0}+k \pi / \iota$. P'oiché:

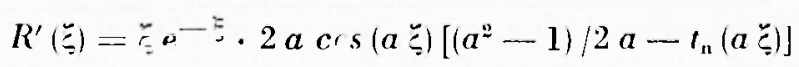

tale derivata $\dot{c}$ positiva nellintervallo $10, \stackrel{,}{,})$, e negli intervalli $\xi_{0}+(2 k-1) \pi / a, \breve{\zeta}_{0}+2 k \pi / \mathrm{a} ; \mathrm{k}=1,2, \ldots$, negativa negli intervalli

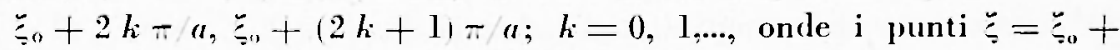
$+(2 k-1) \pi / a$ sono per $R\left(\xi_{\xi}\right)$ di minimo, i punti: $\Xi=\check{\Xi}_{0}+2 k \pi / a$ sono di massimo relativi: (Fig. 2e):

$$
\begin{array}{r}
R_{\max } \cdot=\left[\left(1+\check{\zeta}_{0}+2 k \pi / a\right) \cos a \check{\zeta}_{0}+\left(a \check{\zeta}_{0}+2 k \pi\right) \operatorname{sen} a \check{\zeta}_{0}\right] \\
\exp \cdot\left[-\xi_{0}+2 k \pi / a\right]>0 \\
R_{\min } \cdot=-\left[\left(1+\check{\zeta}_{0}+(2 k-1) \pi / a\right) \cos a \check{\zeta}_{0}+\left(a \check{\zeta}_{0}+(2 k-1) \pi \mid a\right) \operatorname{sen} a \check{\zeta}_{0}\right] \\
\exp \cdot\left[-\xi_{0}-(2 k-1) \pi / a\right)<0
\end{array}
$$

e decrescono col crescere di $k$. 
Il modulo della ridotta radiale $M\left(E_{\mathrm{r}} / E_{\mathrm{rc}}\right)$, per $t=T / 4$, in funzione della costante dielettrica $\grave{e}$ una funzione crescente di $\Xi$.

$$
\begin{aligned}
& M(\varepsilon)=e^{-\xi}\left[(1+\xi)^{2}+a^{2} \xi^{2}\right]^{1 / 2} \\
& M(0)=e^{-\xi}(1+2 \xi+2 \xi)^{1 / 2}, M(\infty)=\infty, \\
& \left.M^{\prime}(\varepsilon)=\omega^{2} \xi^{2} \varepsilon e^{-\xi}\left[4 \cdot \pi^{2} \sigma^{2}\right)(1+\xi)^{2}+a^{2} \xi^{2} !\right]^{-1} \\
& M^{\prime}(\varepsilon)=0, \varepsilon=0 ; \text { per } \varepsilon>0, M^{\prime}(\varepsilon>0, M(\varepsilon) \text { crescente. }
\end{aligned}
$$

Poiché: $M^{\prime \prime}(\varepsilon)=\left(4 \pi^{2} \sigma^{2}\right)^{-1}()^{2} \xi^{2} e^{-\xi}\left(1+2 \xi+2 \xi^{2}\right) \cdot\left[(1+\zeta)^{2}+a^{2} \xi^{2}\right]^{-312}$

è sempre positiva qualunque sia $\Xi$, il grafico di $M(\xi)$ volge la concavità verso l'alto (v. Fig. 3):

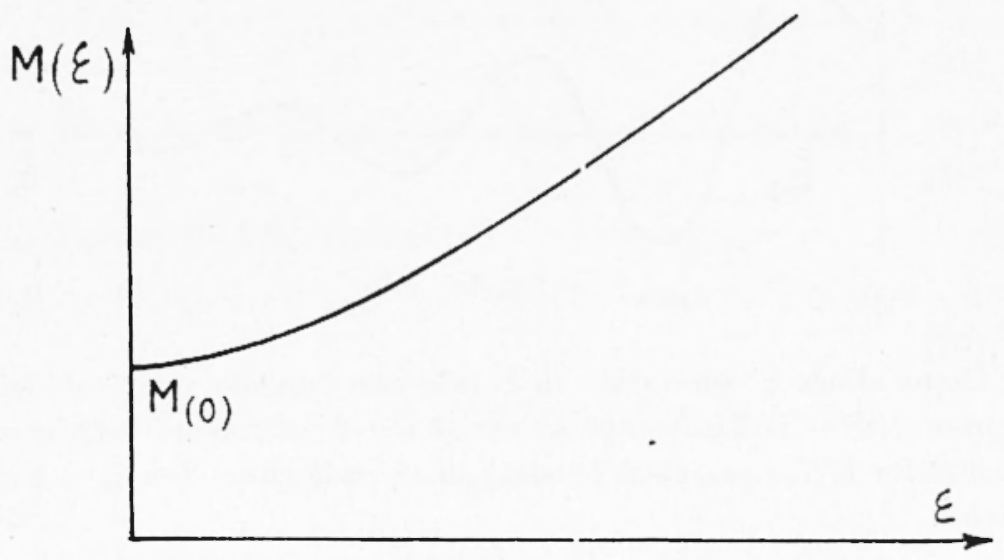

Fig. 3

Il che è confermato indirettamente dalle ampiezze dei "fattori di correzione " dei campi statici: $A=(1+\gamma r)$. ${ }^{-\% r}$ prr $E_{r}, B=$ $\left(1+\gamma r+\gamma^{2} r^{2}\right)$. $e^{-\because}$, per $E_{\rho}$ in funzione di $\xi_{\text {in }}$ un diagramma di J. R. Wait [6]: i fasci di curve $A, B$ indicano un'attenuazione massima se $(f \Sigma / \sigma)$ è zero, che diminuisce con l'aumentare di tale parametro.

Il modulo di $M\left[E_{\mathrm{r}} E_{\mathrm{rc}}\right]$, al variare invece di $\Xi$, comporta: $M(o)=$ $1, M(\infty)=o, M^{\prime}(\xi)$ derivata che s'annulla se:

$$
\zeta_{1}=0, \zeta_{2}=\left(a^{2}-1\right) /\left(a^{2}+1\right) \text {. }
$$

Poiclıé:

$$
M^{\prime}(\xi)=-\left(a^{2}+1\right) \xi e^{-\xi} /\left[(1+\xi)^{2}+a^{2} \xi_{2}^{2}\right]_{1 / 2},
$$


per: $0<\varepsilon<\xi_{\text {s. }} . M^{\prime}(\xi)>0, M(\xi)$ crescente, per $\xi_{0}<\xi<\infty, M^{\prime}(\xi)<0, M(\xi)$ decrescente, onde per $\xi-0, M(\xi)$ ha un minimo relativo di valore uno, per $\xi=\xi_{\text {, }}, M(\xi)$ ( ha un massimo ): $a\left(x p-\left(a^{2}-1\right) /\left(a^{2}+1\right)\right.$.

11 grafico $[M(\xi), \xi]$ risulta molto simile a quello di Fig. la, salvo una diversa decrescenza dopo il massimo, ma su cui la discussione dettagliata è rimandata altrove.

La fase del modulo di $M(\varepsilon, \zeta \zeta$, al variare di $\varepsilon$, è derivabile da:

$\operatorname{tg} \varphi(\varepsilon)=[a \xi \operatorname{sen}(a \xi)+(1+\xi) \cos (1 \xi)] /[(1+\xi) \operatorname{son}(a \xi)-a \xi \cos (a \xi)]$,

$$
a^{2}=1+\omega^{2} \Sigma^{2} / 4 \pi^{2} \sigma^{2}
$$

funzione crescente di $\Sigma$. Detta tangente $\dot{e}$ definita qualunque sia $a \geqslant 1$, salvo che per valori di $a$ per cui è: $(1+\xi) \operatorname{sen}(a \xi)=a \zeta \cos (a \xi)$; cioè non potendo tale espressione annullarsi quando sia : $\cos (a \zeta)=0$, per i valori di a per cui : $\operatorname{tg}(a \xi)=\xi a /(1+\xi)$, che sono le ascisse dei punti d'intersezione dei grafici delle funzioni: $a \xi /(1+\xi)$, e $\lg (a)$ қे).

Poiché: $d \operatorname{tg} \varphi / d a<o, \operatorname{tg} \varphi$ è decrescente. Detto $a_{\mathrm{K}}$ il generico punto in cui s'annulla il denominatore di $t g$ o (che è punto d'infinito di $\operatorname{tg}$ '? stessal si ha:

$$
\lim _{a \rightarrow a_{K}} \operatorname{tg} \varphi /=-\infty \quad ; \quad \lim _{a \rightarrow-a_{K}} \operatorname{tg} \varphi=+\infty
$$

Il diagramma $[\stackrel{p}{a}, a]$ darà valori definiti a meno di multipli di $\pi$. Analogo comportamento si ha per la fase del modulo derivabile da $\operatorname{tg} \varphi(\xi)$, funzione definita per tutti i valori di $\zeta$ salvo quelli per cui è: $\lg (a \xi)=\xi /(1+\xi)$, ascisse dei punti d'intersezione dei grafici delle 2 funzioni a $1^{\circ}$ e $2^{\prime \prime}$ membro dell'equazione precedente. La derivata di $\operatorname{tg} \varphi(\xi)$ rispetto a $\zeta$ è negativa, si annulla per $\zeta=0$ e per $\xi=$ $-2 /\left(a^{2}+1\right)$. Per $\breve{\zeta}=0$ si annullano sia il denominatore della funzione che quello della derivata, quindi nel campo $(0<\xi<\infty)$ la funzione non ha né massimi, né minimi relativi, e a causa del segno della derivata la $\lg \varphi(\zeta)$ risulta decrescente.

Di conseguenza, detti $\xi_{-1}, \zeta_{\vartheta}, . . \zeta_{K}, .$. i punti di non definizione della funzione, per la decrescenza della funzione stessa, si deduce: non esiste il limite per $\zeta \rightarrow \infty$ di $\operatorname{tg} \varphi(\xi)$,

$$
\lim _{\xi \rightarrow \xi_{K^{-}}} \operatorname{tg} \varphi\left(\xi_{0}\right)=-\infty, \lim _{\xi \rightarrow \xi_{K^{+}}} \operatorname{tg} \varphi(\xi)=+\infty .
$$


II grafico $[\varphi(\xi), \xi]$ è simile al precedente $[\varphi(\varepsilon), a]$ e dà pur esso valori definiti a meno di multipli di $\pi$.

Considerato che le velocita di trasmissione nel suolo sono: $v=c / n$, $v^{*}=c(f / \sigma)^{1 / 2}$, nelle due situazioni: $E_{\mathrm{r}}(\varepsilon, \sigma), E_{\mathrm{r}}(\sigma)$, allora il loro rapporto è dato da:

$$
v / v^{*}=(2 \pi \sigma)^{1 / 2} \cdot\left[z+z^{-1} \cdot 4 \pi^{2} \sigma^{*}\right]^{-1 / z} ; z=\varepsilon(1)
$$

tale che:

$$
\left(v / v^{*}\right)_{z \rightarrow-\infty}=\left(v / v^{*}\right)_{z \rightarrow \infty}=0,\left(v / v^{*}\right)^{1}=-(2 \pi \sigma)^{1 / 2} \cdot f(\sigma, z)
$$

Il massimo di: $\left(v / v^{*}\right)$, cade per $z=2 \pi \sigma:\left(v / v^{*}\right)_{\max }=\frac{1}{\sqrt{2}}$, quindi è costantemente: $v<v^{*}$.

4" proprietà: Ia incidenza di $\varepsilon(()$,$) sul fattore di propagazione$ (nel senso qui indicatol si ripercuote perciò oltre che sull'entità del campo elettrico, che s'incrementa, sull'ulteriore riduzione delle velocità di trasmissione delle o.e.m. nei suoli f grià ridotte con le hasse frequenze), ad ordini di grandezza di velocità sismiche. Si creano con ciò situazioni ovviamente vantagrgiose per la prospezione geoelettrica, salvo che la profondita di penetrazione della ca. nel terreno diminuisce in funzione del coefficiente: $\left(1+i(s) \sigma^{-1}\right)^{1 / 2}$.

\$ 4. Rimuoviamo infine l'approssimazione della dipolarita, e calcoliamo il campo elettrico di un bipolo $(-a,+a$, posto lungo $x$, in punti $P(\xi, \eta)$ alla distanza: $r=\left[(\zeta-x)^{2}+r_{i}^{2}\right]^{t / 2}$ dall'eccitatore: $(a<\xi<+\infty)$ : (da porre attenzione al nuovo sionificato qui attribuito alla $\xi$ ).

L'integrale indefinito della [2] rispetto ad $x, 1 \leqslant 0 r_{1}=0$, sara:

$$
\begin{aligned}
& \Sigma(\xi)=(2 \pi \sigma)^{-1} \cdot\left[(\xi-x)^{-2}-\frac{3}{4} \xi^{2}(\xi-x)^{-4}-e^{-\gamma(\xi-x)} \cdot(\xi-x)^{-2} / 2-\right. \\
& -\frac{\gamma}{2}(\xi-x)^{-1} e^{-\gamma(\xi-x)}+\frac{\gamma^{2}}{2}\left|(\xi-x)^{-1} e^{\gamma(\xi-x)} d x\right|,[38]
\end{aligned}
$$


$\gamma=2 \pi(1+i) \sqrt{\prime \sigma l}=\alpha(1+i), \gamma^{2}=2 i \alpha^{2}, \alpha=2 \pi / \overline{\sigma l},(\bar{z}$ qui indica una ascissa), quindi :

$$
\begin{aligned}
& R[\Sigma(\xi)]=(2 \pi \sigma)^{-1}\left[\Phi_{1}(a, \xi, \alpha)+\alpha^{2} \int_{-a}^{+a}(\xi-x)^{-1} e^{-\alpha(\xi-x)} \operatorname{sen} \alpha(\xi-x) d x\right] \\
& J[\Sigma(\xi)]=(2 \pi \sigma)^{-1}\left[\left(D_{2}(a, \xi, \alpha)+x^{2} \int_{-1}^{+a}(\xi-x)^{-1} e^{-\alpha(\xi-x)} \cos \alpha(\xi-x) d x \mid\right.\right. \\
& \Phi_{i}=(\xi-a)^{-2}-\frac{3}{4} \xi^{2}(\xi-a)^{-4}-\frac{1}{2}(\xi-a)^{-2} e^{-a(\xi-a)} \cos \alpha(\xi-a) \\
& -\frac{1}{2}(\xi-a)^{-1} \alpha e^{-0(\xi-a)}[\cos \alpha(\zeta-a)+\sin \alpha(\xi-a)]-(\xi+a)^{-2}- \\
& -\frac{3}{4} \xi^{2}(\zeta+a)^{-4}+\frac{1}{2}(\zeta+a)^{-2} e^{-\alpha(\xi+a)} \operatorname{ccs} \alpha(\xi+a)+ \\
& +\frac{1}{2}(\xi+a)^{-1} \alpha e^{-(\xi(\xi+a)}[\cos \alpha(\xi+a)+\operatorname{sen} \alpha(\xi+a)] \text {. } \\
& \Phi_{2}=\frac{1}{2}(\xi-a)^{-2} e^{-\alpha(\xi-a)} \operatorname{sen} \alpha(\xi-a) \\
& -\frac{1}{2}(\xi-a)^{-1} \alpha e^{-\alpha(\xi-a)}[\cos \alpha(\xi-a)-\operatorname{sen} \alpha(\xi-a)- \\
& -\frac{1}{2}(\xi+a)^{-\varepsilon} e^{-\alpha(\xi-a)} \operatorname{sen} \alpha(\xi+a)- \\
& -\frac{1}{2}(\xi+a)^{-1} \alpha e^{-\alpha(\xi+a)}\{\cos \alpha(\xi+a)-\operatorname{son} \alpha(\xi+a)]
\end{aligned}
$$

Nell’ipotesi che $(\alpha . r \sim r / \lambda)$, sia molto piccolo, per terreni normali, a hasse frequenze. le precedenti formole si semplificano notevolmente:

$$
\begin{aligned}
&\left.R[\Sigma(\xi)]=R(\xi)=(8 \pi \sigma)^{-1}\right)(\xi+a)^{-1}\left[\xi^{2}-4 a \xi-2 a^{2}\right] \\
&-(\xi-a)^{-1}\left[\xi^{2}+4 a \xi-2 a^{2}\right]-a \alpha^{3} / \pi \sigma_{i}^{\prime}
\end{aligned}
$$$$
R(0)=-\infty, R(\alpha)=-a \alpha^{3} / \pi \sigma, R^{1}(\alpha)=(4 \pi \sigma)^{-1}[\varphi(-a, \xi)-\varphi(a, \xi)]
$$$$
\varphi(\lambda, \xi)=(\xi+\lambda)^{-5} \cdot\left(\xi^{2}-7 \lambda \xi-2 \lambda^{2}\right) ; \varphi(0, \lambda)=\xi^{-3}>0, \varphi(\lambda, \infty)=0
$$$$
\varphi^{\prime}(\lambda, \xi)=6(\xi+\lambda)^{-0} \cdot\left(\lambda^{2}+4 \xi \lambda-2 \xi^{2}\right) ; \varphi^{\prime}(\lambda, s)=0 \text {, se } \lambda=\xi(2+\sqrt{6}) \text {. }
$$ 
Si ha con ciò $\varphi(\xi, \lambda)$ in funzione di $\lambda$, con un max positivo in $\xi \cdot(-2-\sqrt{6})$ e un minimo negativo per $\xi(-2+\sqrt{6})$.

Essendo $\xi>a, \xi\left(-2-\gamma^{-} 6\right)<-a$, allora qualunque sia $\xi$, $\varphi(a, \xi)<\varphi(-a, \xi)$ onde $\varphi(-a, \xi)-\varphi(a, \xi)>0$, quindi : $R^{\prime}(\xi)>0$ qualunque sia $\xi \geqslant a$. Pertanto $R(\xi)$ è crescente al crescere di $\xi$.

Inoltre: $R^{\prime \prime}(\xi)=3 \cdot(4 \pi \sigma)^{-1} \cdot[\psi(a, \xi)-\psi(-a, \xi)]$, il segno di $R^{\prime \prime}(\xi)$ dipende, per ogni $\xi$. da quello dell'incremento di $\psi(\lambda, \xi)$ : $\psi(0, \bar{\xi})=\bar{\xi} ;, \dot{\zeta}, \dot{\zeta}=0, \psi^{\prime}(\lambda, \xi)=8(\xi+\lambda)^{-7} \cdot\left(\lambda^{2}+6 \lambda \xi-2 \xi^{2}\right)$,
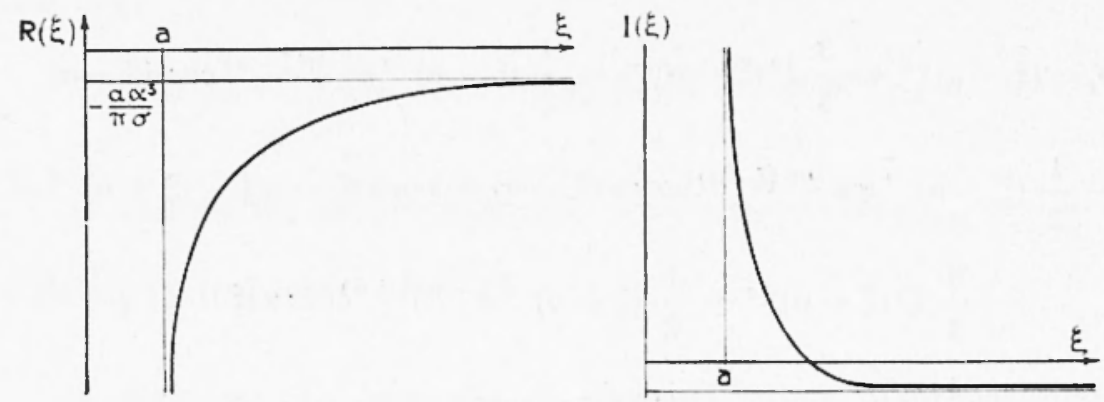

Fig. 4

che $\dot{e}$ zero per $\lambda=(-3-\sqrt{11}) \xi$, e quindi il grafico di offre un max $>0$ in $(-3-\sqrt{11}) \xi$, un minimo $<0$ per: $\xi(-3+\sqrt{11})$

Tenuto conto che essendo $\varepsilon>a,(-3-\sqrt{11}) \varepsilon<-a, \psi(-a, \xi)$ $>\psi(a, \xi)$, quindi : $\psi(a, \xi)-\psi(-a, \xi)<0, R^{\prime \prime}(\vec{\xi})<0$ per ogni $\xi \geqslant a$.

$5^{a}$ proprietà: Pertanto il grafico di $R(E)$ volge la concavitì verso

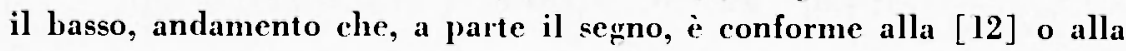
[20]: (Fig. 4a).

La : $I[\unlhd(\xi)]=I(\xi)$, a semplificazioni effettuate risulta :

$$
\begin{gathered}
I(\xi)=(2 \pi \sigma)^{-1} \cdot \alpha^{2}[\log !(\xi+a) /(\xi-a) !-a \alpha] \\
I(a)=+\infty, I(\infty)=-a \alpha^{3} / 2 \pi \sigma \\
\left.I^{\prime}(\xi)=-a \alpha^{2} \mid \pi \sigma\left(\xi^{2}-a^{2}\right)\right]^{-1}<0 \\
I^{\prime \prime}(\xi)=(\pi \sigma)^{-1} a \alpha^{2} \cdot 2 \xi \cdot\left(\xi^{2}-a^{2}\right)^{-2}>0
\end{gathered}
$$

$I(\vec{\xi})$ risulta decrescente e la concavitì della curva (senza flessi), è rivolta verso l'alto: (v. Fig. 4). 
Dopo l'esame del campo hipolare (nelle condizioni espresse) con la distanza dall'emittore, vediamo come esso varia con $\alpha$, con la frequenza, o con la conduttività, ferma restando la distanza.

Dalla formola non ridotta [38] della $R(\alpha)$ si ricava:

$$
\begin{aligned}
R(0)= & (2 \pi \sigma)^{-1}\left\{\frac{1}{2}(\xi-a)^{-2}-\frac{3}{4}(\xi-a)^{-1}\right. \\
& \left.-\left[\frac{1}{2}(\xi+a)^{-2}-\frac{3}{4} \xi^{2}(\xi+a)^{-1}\right]\right\} \\
R(\alpha)= & (2 \pi \sigma)^{-1} ;(\xi-a)^{-2}-\frac{3}{4} \xi^{2}(\xi-a)^{-1} \\
& \left.-\left[(\xi+a)^{-2}-\frac{3}{4} \zeta^{2}(\xi+a)^{-1}\right]\right\} \\
K^{\prime}(\alpha)= & (\tau: \sigma)^{-1} \alpha \cdot \varphi(\alpha), \\
& \int_{-a}^{+a}(\xi-x)^{-1} e^{-\alpha(\xi-x)} s \quad n \alpha(\xi-x) d x
\end{aligned}
$$

Il kegno di $R^{\prime}(\alpha)$, dato che $\left.\alpha>1\right)$, dipende da quello di $\rho(\alpha)$ il che importa di conoscere $p(\alpha)$ al variare di $\alpha: \varphi(0)=\varphi(\infty)=0$,

$$
\begin{aligned}
& \varphi^{\prime}(\alpha)=\alpha^{-1}\left[e^{-\alpha(\xi+a)} \operatorname{sen} \alpha(\xi+a)-e^{-\alpha(\zeta+a)} \operatorname{sen} \alpha(\xi-a)\right] \\
& \varphi^{\prime}(\alpha)=\alpha^{-1}: \psi^{\prime}[a(\xi+a)]-\circlearrowleft[a(\xi-a)] ; \psi(z)=\operatorname{sen} z \cdot-x \\
& \psi(0)=\dot{\psi}(\infty)=0, \psi^{\prime}(z)=(\cos z-\operatorname{sen} z) e^{-z}
\end{aligned}
$$
annulla per $k \pi$.

Ne risulta per $\psi(z)$ un diagramma: $\left[\operatorname{sen} z e^{-z}, z\right]$ sinusoidale morzato, da cui si può risalire a quello $\left[\alpha \cdot \varphi^{\prime}(\alpha)\right]$, poi a $[\alpha, \varphi(\alpha)]$. infine a: $[\alpha, R(\alpha)]$.

$6^{n}$ proprietà: La $R\left(x_{1}\right)$ offre 2 diversi aspetti, oscillante smorzato (con saturazione), se la distanza elettrodica $a$ is piccola rispetto a $\xi$ 
(Fig. 5a), aperiodico (con saturazione) (Fig. 5h), se la distanza elettrodica $\dot{e}$ grande.

Ovviamente queste situazioni si prestano per una più opportuna scelta di frequenza. per un maggior "rendimento " di essa.

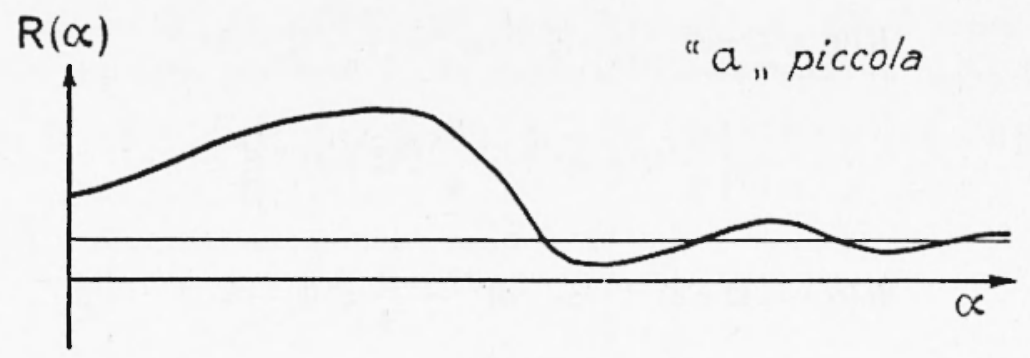

Fig. 5-il

Sia $R(o)$ che $R(\chi)$ possono essere $\geqslant 0$, a seconda i rapporti relativi di $a$ e $\xi$, che dohbiamo precisare.

Posto: $\quad \dot{\psi}(z)=(2 \pi \sigma)^{-1}\left[\frac{1}{2} z^{-z}-\frac{3}{4} \xi^{2} z^{-1}\right]$, la [40] diviene:

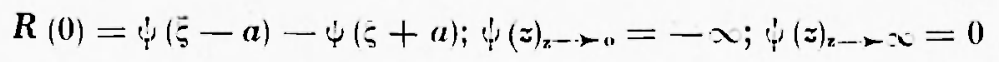

$$
\begin{aligned}
& \dot{\psi}^{\prime}(z)=\left(2 \pi \sigma z^{3}\right)^{-1} \cdot\left(3 \%^{2}-z^{2}\right) ; \bigcup^{\prime}(z)=0,(z=\xi \mid \overline{3}) \text {, }
\end{aligned}
$$

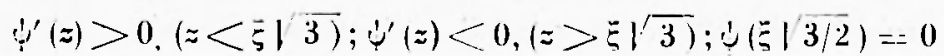

Se: $\xi-a>\xi \sqrt{3},(\xi+a>\xi-a>\xi ! 3)$, si ha, per la decrescenza della funzione: $\psi(\xi-a)-\dot{\zeta}(\bar{\sigma}+a)>0, R(0)>0$; se: $\xi-a<\xi \sqrt{3},(\xi-a<\xi \sqrt{3}$ ), si ha per la crescenza della funzione: ( $)-a)-\psi(\xi+a)<0, R(0)<0$.

$V i$ è uno ed un sol valore di $(\xi-\mu)$, e quindi di $(\xi+\mu)$, per cui $R(o)=o$.

Analogamente: posto $\dot{\psi}^{*}(z)=(2 \pi \sigma)^{-1} \cdot\left(z^{-2}--\frac{3}{4} \xi^{2} z^{-4}\right)$. la [41] assume la forma: $R(\lambda)=\zeta^{*}(\xi-a)-\xi^{*}(\xi+a)$, con $\dot{\zeta}^{*}(z)_{z \rightarrow 0}=-\infty$, $\psi^{*}(z)_{z \rightarrow} \rightarrow \infty=0, \psi^{* \prime}(z)=\left(2 \pi \sigma z^{*}\right)^{-1} \cdot\left(3 \zeta^{-}-2 z^{*}\right), \iota^{* \prime}(z)=0$ per $z=5 / \sqrt{3 / 2}$, $\psi^{\prime \prime}(z)>0$ per: $0<\xi \sqrt{3,2}<0,<0$ per $z>\xi l^{\prime} \overline{3 / 2}$, con lo stesso andamento di $\psi(z)$.

Se: $\xi+a<\xi \sqrt{3 / 2},(\xi-a<\xi \sqrt{3 / 2})$, si ha, per la crescenza della funzione: $\psi(\xi-a)-\psi(\xi+a)<o$, quindi $R(\alpha)<o$; se: 
$\xi-a>\xi \sqrt{3 / 2},(\xi+a>\xi \sqrt{3 / 2})$ si ha per la decrescenza della funzione $\psi(\xi-a)-\varphi(\xi+a)>0, R(x)>0$. Vi è un solo valore di $(\xi-a)$, (e di $\xi+a)$ per cui $R(\infty)=o$.

Così dopo un'introduzione sugli scopi principali di questa "Memoria ", ricordato rapidamente la teoria del Sommerfeld e deduzioni

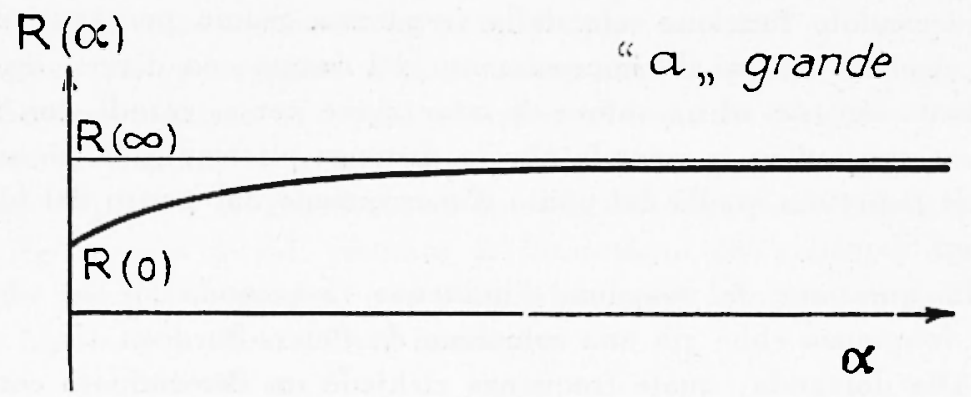

Fig. 5.b

relative al campo elettrico terrestre alla superficie del suolo con le sue principali proprietà, abbiamo costruito i grafici di fig. 1 illustranti i risultati sostanzialmente già conseguiti fin dal 1942 dallo Stefanesco, e dieci anni dopo ripresentati da Yost ed altri, con leggere varianti. Si ì ripercorso questa teoria classica (sia pure ormai insuffciente) per far risaltare alcune deduzioni che permangono tuttora valide, tra cui la formola fondamentale (trovata per altra via dall'Horton' per il calcolo della parte reale della componente radiale del campo elettrico.

Segue una minuta necessaria disamina dell'evoluzione spaziale e temporale delle "componenti ridotte" del campo elettrico, nelle loro parti rispettive reali e immaginarie.

Dopo di che ci siamo posti nelle condizioni dell'Horton, più aderenti alla sperimentazione procedendo con analisi analoghe alle precedenti.

In seguito partendo dalle formole generali Smith-Horton-Lewis abbiamo sviluppata quest'ultima teoria, estendendo l'ipotesi relative al rapporto $(\sigma / \omega \equiv \geq 1)$.

Da porre attenzione al modo d'interpretare della nuova alternativa: $\sigma_{1} \omega s<1$, normalmente giustificata tale per 1 altissime, mentre impiegando w basse, si è costretti assumere, (per la validità della disuguaglianza , altissime costanti dielettriche, dell'ordine riconosciuto dall'Evjen. 
Rimuoviamo infine l'approssimazione della dipolarità, introducendo emittori bipolari.

Calcoli laboriosi hanno pur condotto a stabilire comportamenti di campo con la distanza del bipolo, analoghi a quelli aperiodici del dipolo a bassissima frequenza.

L'esame del campo bipolare $\grave{e}$ stato ulteriormente approfondito, considerandolo funzione solo della frequenza. Siamo pervenuti a diversi risultati tra cui il riconoscimento del campo con diversi aspetti, oscillante intorno ad un valore di saturazione per $\omega$ grandi, con massimo, o aperiodico, a seconda che la distanza elettrodica ì piccola o grande rispetto a quella del punto d'osservazione dal centro del bipolo (v. fig. 5).

La questione del massimo d'influenza (a seconda $\sigma$ ), col variare della frequenza ebbe già una soluzione da Peters-Bardeen $1 \%$

Alla domanda: quale frequenza richiede un determinato conduttore spaziale per provocare la massima influenza e.m. in superficie?, questi AA. hanno potuto rispondere determinandola conforme ai nostri risultati.

Con emittore dipolare, si pronuncia un ottimo di frequenza in termini di profondità d'investigazione e conduttività del mezzo, mentre con emittore bipolare (distanza elettrodica grande) si ha (nelle condizioni esaminate), una saturazione aperiodica da un massimo iniziale d'influenza a frequenza nulla.

Cagliari - Facoltà d'Ingegneria dell Lniversità - Maggio 1954.

\section{RIASSLNTO}

L'A. si sofferma dapprima rapidamente sulla teoria classica del Sommerfeld del dipolo orizzontale oscillante, posto alla superficie d'un suolo omogeneo, (teoria che trascura la costante dielettrica a causa delle medie frequenze adoperate), presentando i risultati fondamentali con grafici e tabelle dimpiego nella prospezione elettrica del sottosuolo.

Si mostra l'evoluzione delle acomponenti ridotte s radiali o tangenziale del campo e.m. terrestre nelle loro rispettive parti reale $e$ immaginaria, in funzione della * distanza numerica *

Si ricava una formola approssimatn che da la parte reale della componente radiale (quella generalmente che si misura sul terreno), coincidente con una data dallHorton, seguendo un diverso indirizzo deduttivo. 
Nella $2^{a}$ parte l'A. discute la teoria Horton-Lewis vertente sullargomento o mostratasi più aderente alla realtà sperimentale, con relativo esame dettagliato.

Nella $3^{a}$ parte l'A. sviluppa una teoria, la quale tenendo presente $i$ piü recenti studi di Evjen, conferisce alla costante dielettrica dei suoli la sua dipendenza dalla bassa frequenza.

Con tali presupposti deduce come alle bassissime frequenze si debba incrementare il campo elettrico alternativo e ridurre notevolmente la velocità di trasmissione delle onde elettromagnetiche del suolo, allordine di grandezza delle velocità delle onde sismiche.

Nella parte $4^{a} l ' A$. rimuove la limitazione dell'emittore dipolare dando formole e grafici, con particolareggiate discussioni dell'emittore bipolare in determinate condizioni.

\section{$S U M M A R Y$}

The author first treats rather rapidly the classical theory of Sommerfeld of the horizontal oscillating dipole, placed at the surface of a homogeneous soil (a theory which, because of the mean frequencies adopted, neglects the dielectric constant), presenting the fundamental results with graplis and tables for use in electric prospecting of the under soil.

One shou's the behavior of the radial and tangential "reduced components of the earth's e.m. field in their respective real and imaginary parts, as a function of the "numerical distance".

An approximate formula which gives the real part of the radial component (that which one generally measures at the earth surface) ha: been found which agrees with that given by Horton following another deductive line of argument.

In the second part, the theory of Horton-Lewis bearing on this argument is discussed, and is shown to be closer to the experimental reality after a detailed examination.

I nthe third part, the author develops a theory, which, heeping in mind the most recent studies of Evjen, shous the dependence of the dielectric constant of the soil on the frequency.

With such suppositions, it is deduced houc, at the very lowest frequencies, one must increase the alternating electric field and reduce notably the transmission velocity of e.m. waves of the soil, to the order of magnitude of the seismic wave velocity. 
In part four, the author removes the limitation of the dipole emittor, giving formulae and graphs, with detailed discussions of the bipole oscillator.

\section{BIBLIOGRAFIA}

(1) Bellu 1Gi A., Sui canpt elettromagnetici a bassa frequenza. Rivista di Geofisica Appl. 1952, Milano.

(-) Bralley Lewis W., Electric Field of a oscillating Dipole. Geophysik, 1916.

(3) Honton C. W., On the use of e.m. Waves in Geoph. Prospecting. Geophysics, 1946.

(t) Stefanescu S. S., Das elehtromagnetische Normalfeld des wangerechten Nieder/requenz dipols. Beitr. zur angewand. Geophysik, 1912, B. 9.

(5) Wouf A., Electric Field of a Oscillating Dipole on the surface of a Two Layer Earth. Geophysies, 1946.

(6) Watt J. R., A Nole on Dipole radiation in a Conducting Medium. Geophysic's, 1952, n. 4 .

(5) Peters L. Barneax J., Some Aspect of Electrical Prospecting applied in Locating Oil Structures. Geopli., 1931. 